This article is licensed under the Creative Commons Attribution-NonCommercial 4.0 International License (CC BY-NC) (http://www.karger.com/Services/OpenAccessLicense). Usage and distribution for commercial purposes requires written permission.

\title{
Use of a Perioperative Web-Based Exercise Program for a Patient with Barrett's Carcinoma Scheduled for Esophagectomy
}

\author{
Barlo Hillen $^{a} \quad$ Perikles Simon $^{a} \quad$ Peter P. Grimminger ${ }^{b} \quad$ Ines Gockel ${ }^{c}$ \\ Daniel Pfirrmann ${ }^{\text {a }}$ \\ aDepartment of Sports Medicine, Disease Prevention and Rehabilitation, Institute of \\ Sports Science, Johannes Gutenberg University Mainz, Mainz, Germany; ${ }^{\text {bDepartment of }}$ \\ General, Visceral and Transplant Surgery, Johannes Gutenberg University Medical Center \\ Mainz, Mainz, Germany; 'Department of Visceral, Transplant, Thoracic and Vascular \\ Surgery, University Hospital of Leipzig, Leipzig, Germany
}

\section{Keywords}

Esophageal cancer - Barrett's carcinoma - Preconditioning · Exercise · Perioperative care · Prehabilitation · Web-based

\begin{abstract}
A poor preoperative functional capacity increases the perioperative risk. Therefore, a webbased exercise approach has been initiated for a careful supervision and individual support of patients during their perioperative cancer therapy. Here, we present the data of a 57-year-old patient, scheduled for esophagectomy. Beside a five-week neoadjuvant chemoradiotherapy (CRT), the patient performed 10 weeks of a web-based prehabilitation exercise training (preconditioning) and continued the exercise program for 14 weeks after surgery. The patient performed 42 of 44 recommended training sessions in the preconditioning period in his home
\end{abstract}


environment. This corresponds to a mean of $131( \pm 38)$ min of total training per week. The patient performed 52 of 48 recommended training sessions in the postconditioning period. This corresponds to a mean of $165( \pm 87)$ min of total training per week. The patient could maintain his functional capacity during CRT and enhanced his submaximal performance level to the end of the intervention. The patient's adherence to the program was noteworthy. The most important features of this approach are the personalized design and the flexible scheduling precisely in tune with the patient's needs, concerns, and therapy related downtimes. Thus, the patient nearly fulfilled the exercise guidelines at a sufficient exercise intensity even during the neoadjuvant therapy. The preconditional exercise prevented a presumed CRT-induced and tumor-associated loss of functional capacity. The postoperative exercise led to a structured and adequate return into regular physical activities after surgery. Finally, this approach complements a comprehensive clinical care, in a health-promoting, cost-effective, and patientsafely manner.

(C) 2019 The Author(s)

Published by S. Karger AG, Basel

\section{Introduction}

Tumor resection is the only curative treatment for esophageal cancer. The surgical procedure and the mostly accompanied neoadjuvant chemoradiotherapy (CRT) are often associated with a reduction in cardiopulmonary function and a high level of morbidity $[1,2]$. Preoperative functional capacity is an important predictor for the postoperative prognosis [2-4]. Prehabilitation, correctly implemented, improves functional capacity and reduces postoperative complications [5, 6]. Relevant clinical effects of exercise interventions have been proven over all phases of the oncological treatment [7]. These exercise interventions were also feasible for patients undergoing CRT [8]. However, a limited period of time between diagnosis and resection usually leads to postpone exercise therapy to the postoperative period [9]. Since a poor preoperative functional capacity increases the perioperative risk, patients should be additionally and ideally transferred to an individualized exercise prehabilitation program. A group-based, supervised exercise program, which should take place several times a week, in order to achieve positive effects, is complex to implement [10]. These programs are limited to a clinical center and are not regularly accessible to patients from rural areas. Furthermore, inpatient programs are costly, and the adherence is a crucial issue [11]. Prehabilitation and postoperative exercise programs need to be: firstly, tailored to individual needs and immediately adaptable to new circumstances, secondly, dynamic with a progressive increase in the exercise recommendations, thirdly, feasible to enhance patients' adherence, fourthly, controlled by a trained professional and fifthly, accessible at any time [3]. In response to these challenges, a web-based exercise approach has been initiated for careful supervision and individual support of patients in their living environment during their perioperative cancer therapy [12]. 


\section{Case Reports in Oncology}

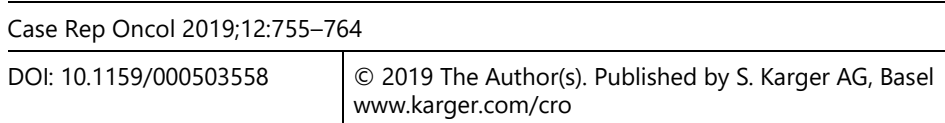

Hillen et al.: Use of a Perioperative Web-Based Exercise Program for a Patient with Barrett's Carcinoma Scheduled for Esophagectomy

\section{Case Presentation}

The presented case was a participant in the internet-based perioperative exercise program (iPEP-study); (Trial Registration ClinicalTrials.gov: NCT02478996). Patients in the intervention group (IG) perform an individualized exercise program until surgery (between 8 and 12 weeks), and after surgery for another period of 12 weeks [12]. Here, we present the data of a 57 year-old patient, scheduled for esophagectomy, without athletic experience. The man was diagnosed with esophageal carcinoma (stage: cT3 cN + cM0; histology: adenocarcinoma; secondary diagnosis: depressive disorder). Beside a five-week neoadjuvant CRT (radiotherapy: ZV1A: 3DCRT, photons; 41.4 Gy; $5 \times 1.8$ Gy/week; chemotherapy: $5 \times$ Paclitaxel 50 $\mathrm{mg} / \mathrm{m}^{2} \mathrm{KOF}+$ Carboplatin AUC2) according to the CROSS regimen [13] he performed 10 weeks of the web-based prehabilitation exercise training (preconditioning). He finished the tailored exercise program (postconditioning) following oncologic esophagectomy and 11 weeks of inpatient rehabilitation after 36 weeks (Fig. 1).

At the beginning of the web-based exercise program, the patient was registered on our website, and he obtained the training materials, including resistance bands (Pinofit ${ }^{\circledR}$ ) and a heart rate monitor (FT1, Polar ${ }^{\circledR}$ ). The website enabled the patient to receive individual exercise recommendations from a sports therapist, to download exercise descriptions as PDF and video files, and to interact with other patients. The study participant was thoroughly tested three times in our department (T0-T2). To each measuring point, anthropometric data, first second of forced expiration $\left(\mathrm{FEV}_{1}\right)$ and forced vital capacity (FVC) were assessed (Bodybox 5500 , Medisoft), functional capacity (maximum oxygen uptake $\left(\mathrm{VO}_{2 \mathrm{max}}\right)$, Ventilation, individual anaerobic threshold, and Wattmax) were measured by cardiopulmonary exercise testing (CPET) (treadmill: Saturn, HP Cosmos ${ }^{\circledR}$; Ergostick, Geratherm ${ }^{\circledR}$; ECG: ECGpro, AmedTec ${ }^{\circledR}$ ), including blood lactate analyses (EKF diagnostics ${ }^{\circledR}$; Winlactat 3.0 Mesics GmbH). Further, the dysphagia score, the nutritional risk score, and the stress metabolism score were evaluated. The subjective evaluation of the program content was additionally assessed at the end of the study (Table 2). The first test was before the preconditioning (T0), the second one was 3 days before surgery (T1) and the third was after 14 weeks of postconditioning (T2); (Fig. 1). A modified treadmill walking protocol was chosen for the CPET [12]. During the intervention period, every Monday the sports therapist sent a message with adapted exercise recommendations for the entire week (via Email). The patient was able to decide by himself, when to perform the training during the current week. He was encouraged to perform these exercises, rate the demanded program, based on perceived exertion (RPE; 0-10), and perceived discomfort (RPD; 0-10), and to record the average heartrate to the end of the week [12] (Fig. 2).

\section{Results}

The preconditioning exercise program lasted about ten weeks. CRT was implemented from week two until week six. The patient performed 42 (26 endurance exercise sessions (EE) \& 16 resistance exercise (RE) sessions) of 44 recommended training sessions in the preconditioning period. This corresponds to a mean of $131( \pm 38)$ min of total training $(67 \mathrm{~min}$ endurance exercise; 64 min resistance exercise) per week. The number of exercise recommen- 
dations was progressively increased each month [12]. The patient performed five to seven training sessions per week during the last six weeks before the hospitalization.

The mean RPE value was $7( \pm 2)$ for both exercise types - EE and RE. The mean heart rate (HR) was $141( \pm 2)$ beats/min during EE. This heart rate corresponded to a sufficient training intensity according to his individual anaerobic threshold (IAT). The RPE and the RPD changed conspicuously during CRT and two weeks after the CRT. Whereas the prescribed intensity of EE did not change, the RPE values increased from 5 to 8.5 and reached the maximum of 9.5 in week seven (one week after the CRT). In the same week, the RPD value increased once up to 5. Similarly, the RPE- and RPD values were also higher in RE with the start of CRT. The RPE values increased from 6 to 8.5 and reached their maximum in week 7 and 8, two weeks after CRT. The RPD value increased up to 5 in week 7 and 8 . According to its own statement, the increased RPD value came about by dizziness and nausea and could be further influenced by a present acute gastritis. Contrastingly to EE, the RPD value in RE increased moderately up to 2.5 in week 4, 5 and 6 during CRT. This reported discomfort resulted from chest pain. The information about the surgery and the postoperative complications are listed in Table 1. After the esophagectomy, the patient spent three weeks in the hospital.

After hospital discharge, the patient had five weeks of convalescence followed by three weeks of inpatient rehabilitation. Afterwards, 14 weeks of postconditioning exercise was conducted. The patient performed 52 (31 EE and $21 \mathrm{RE}$ ) of 48 recommended training sessions in the post-conditioning period. This corresponds to a mean of $165( \pm 87) \mathrm{min}$ of total training (95 min EE; 70 min RE) per week. The mean HR was $129( \pm 4)$ beats/min during EE.

The mean RPE was $5( \pm 1)$ for EE and $6( \pm 1)$ for RE, whereas he reported no discomfort (RPD) at all. Two weeks of training interruptions were due to holidays and a cold. The patient reported that he could not reach the demanded HR during endurance training in the first week of the post-conditioning period without giving reasons, but also reported a lower RPE value.

Consequently, the recommendations were immediately adjusted by the sports scientist. The detailed training evaluation is shown in Table 2. According to the subjective statement, the intervention was feasible and helpful for him. The patient used the website regularly and logged in for 70 times and 237 min during the entire course of the intervention.

Of these, 40 Log-ins and 191 min were in the preconditioning period, and 30 Log-ins and $46 \mathrm{~min}$ in the postconditioning period. The physiological changes of T0, T1, and T2 are shown in Table 2 and the blood lactate curve within the IAT is illustrated in Figure 3. Despite CRT, all parameters could be maintained at the same level from T0 to T1. From T1 to T2, a weight loss of $9.6 \mathrm{~kg}$ (90.2-80.6 kg) could be observed. FEV1 decreased from 101-92 \%pred, $\mathrm{VE}_{\max }$ (L/min) decreased from 87 to $78 \mathrm{~L} / \mathrm{min}$, and $\mathrm{VO}_{2}$ max slightly decreased from 23.2 to 22.2 $\mathrm{mL} / \mathrm{min} / \mathrm{kg}$. In the maximum endurance test, the patient completed five increments of the protocol and reached a Watt/ $\mathrm{kg}_{\max }$ of 1.2 to all three measuring time points. The IAT performance increased from 0.9 to 1.1 Watt (Fig. 3).

\section{Discussion}

The present case report demonstrates, that the cost- and time effective, personalized, web-based exercise pre- and postconditioning intervention was tolerable, safe, and helpful for the patient. The patient's adherence to the program was noteworthy. 
The 57 year-old patient reported no difficulties or complications in using the webpage. Thus, the patient was $100 \%$ compliant and uploaded all exercise protocols in time. The regular communication through the webpage was essential, due to unpredictable changes in the health situation and the immediate and dynamic adaptions of the exercise recommendations by the sports therapist. CRT without accompanied exercise therapy causes a clinically significant decrease in $\mathrm{VO}_{2}$ peak and anaerobic threshold (AT) [14]. Further, O'Neill et al. [4] observed that in the first three months post-resection, the $\mathrm{VO}_{2}$ max and the AT are reduced in esophagogastric patients. Contrastingly, the functional capacity of our participant, expressed as $\mathrm{VO}_{2 \max }$ and $\mathrm{Watt}_{\max }$, could be maintained during the entire treatment process.

In current exercise guidelines for cancer patients, it has been recommended that patients should perform "150 min of moderate-intensity aerobic exercise spread over 3-5 days and resistance training at least two days per week" [15]. Resistance sessions should involve "major muscle groups 2-3 days per week (8-10 muscle groups, 8-10 repetitions, two sets), on an ongoing basis as a part of their life." Pre-exercise assessment should be performed and exercise should be conducted in a group or supervised setting [15]. Our concept enabled the patient in the preoperative waiting period, to perform on average, $131 \mathrm{~min} /$ week, $3-5$ session/week (23 endurance; $1-2$ resistance). Thus, the patient nearly fulfilled the exercise guidelines at a sufficient training intensity - even during the neoadjuvant therapy. We implemented pre-exercise assessment by CPET, in order to figure out the optimal individual exercise dosage and for the exclusion of cardiopulmonary limitations and contraindications. For this web-based approach, CPET is an obligatory element in order to prescribe exercise to a sufficient extent and to protect the participant from overload.

Minella et al. [6] examined a structured preoperative exercise intervention, which was feasible, safe, and efficacious in preventing a decrease of functional capacity pre-and post-surgery. This research group observed an increase of functional capacity measured by the 6-minute walking distance (6 MWD) compared to a control group [6]. Even though this assessment is less sensitive, it illustrates the probable differences between non-exercising and exercising patients very well. The prescribed exercises were comparable to our intervention. However, there was only one supervised session per week with additional phone calls for further support [6].

Recently, Singh et al. [8] reported that ten weeks of combined resistance and aerobic exercise during CRT were feasible and improved muscle strength in patients with rectal cancer. In contrast to our program, mainly machine supported resistance training was applied [8]. It seems reasonable to suppose, that machine supported training is more effective than a simple resistance band training. However, it contains demanding procedures for patients and the supporting team. In particular, hospital-based programs are costly and have a high degree of organizational effort [11]. A group-based, supervised exercise program, which should take place several times a week, is therefore difficult to implement $[9,10]$. In addition, these programs are limited to a clinical center and are not regularly accessible to patients from rural areas. An advantage of our approach is the easy and flexible integration of the exercise program into the home environment. Despite the distance, our patient felt safe and well supported during the exercise units.

In the guidelines for perioperative care in esophagectomy, it has been suggested that the value of prehabilitation is at least equivalent to the importance of postoperative programs [16]. According to the guidelines, we could clearly show that preconditioning leads to a 


\section{Case Reports in Oncology}

Case Rep Oncol 2019;12:755-764

DOI: $10.1159 / 000503558$

(C) 2019 The Author(s). Published by S. Karger AG, Base www.karger.com/cro

Hillen et al: Use of a Perioperative Web-Based Exercise Program for a Patient with Barrett's Carcinoma Scheduled for Esophagectomy

sustained functional capacity during and after CRT. In the postconditioning period, we enabled the patient to return to regular physical activity. Treatment related changes and, thus, varying demands and conditions require a tailored exercise approach, throughout all phases of care, in order to individually promote and protect the patient. This case report does not demonstrate how the functional capacity was changed immediately after surgery. Additionally, our study design prevents an assessment of only the post-conditioning program, due to the time gap between T1 ( 3 days before surgery) and T2 (25 weeks after surgery). The results of T2 were influenced by various factors such as surgery, stationary rehabilitation, and the postconditioning program. A CPET after surgery and ideally after the stationary rehabilitation would be necessary to cope with these issues. However, a CPET immediately after surgery is not feasible, due to the patients' acute condition. The amount of weekly performed exercises, as well as the RPE and the RPD, were self-reported by the patient. A measurement of the homebased resistance band exercise was not performed. Strength exercise in this study should rather be seen as a complementary aspect in the exercise program, which is particularly difficult to control in the patient's home environment.

\section{Conclusion}

The personalized, web-based exercise intervention was feasible and helpful for a patient with Barrett's carcinoma scheduled for esophagectomy. The preconditional exercise prevented a presumed CRT-induced loss of functional capacity. The postoperative exercise led to an adequately adapted training regime after surgery and a structured return into regular physical activity. Moreover, the patient's adherence was noteworthy during the preconditioning period, including CRT, and during the postconditioning period. Close patient contact is required to regularly motivate the patient to exercise, to solve exercise related problems and to adjust the exercise program. Finally, this approach complements a comprehensive clinical care, in a health-promoting, cost-effective and patient-safely way.

\section{Acknowledgement}

We thank the Barrett-Initiative e.V. for funding of the iPEP-study.

\section{Statement of Ethics}

The research was conducted ethically in accordance with the World Medical Association Declaration of Helsinki. The subject has given his written informed consent to publish his case.

\section{Disclosure Statement}

The authors have no conflicts of interest to declare. 


\section{Funding Sources}

The Barrett-Initiative e.V. funds the iPEP-Study. The Barrett-Initiative e.V. is a non-profit organization with the aim, to analyze the causes and pathophysiology of Barrett's esophagus and carcinoma. It is an independent organization, and its steering committee decides independently, which Barrett-projects and research are funded. Data collection, analysis, interpretation and writing the manuscript can be supported by the Barrett-Initiative e.V.

\section{Author Contributions}

IG, PS, and DP developed the study concept. DP designed the online tool. PS, and DP designed the exercise components. All authors contributed to the conception, analysis, and interpretation of the manuscript. BH and DP drafted the manuscript. All authors read and approved the final document.

\section{References}

1 Sinclair RC, Phillips AW, Navidi M, Griffin SM, Snowden CP. Pre-operative variables including fitness associated with complications after oesophagectomy. Anaesthesia. 2017 Dec;72(12):1501-7.

2 Bosch DJ, Muijs CT, Mul VE, Beukema JC, Hospers GA, Burgerhof JG, et al. Impact of neoadjuvant chemoradiotherapy on postoperative course after curative-intent transthoracic esophagectomy in esophageal cancer patients. Ann Surg Oncol. 2014 Feb;21(2):605-11.

3 Pfirrmann D, Simon P, Mehdorn M, Hänsig M, Stehr S, Selig L, et al. [Preconditioning prior to visceral oncological surgery: A paradigm shift in visceral surgery?]. Chirurg. 2018 Nov;89(11):896-902.

4 O'Neill L, Moran J, Guinan EM, Reynolds JV, Hussey J. Physical decline and its implications in the management of oesophageal and gastric cancer: a systematic review. J Cancer Surviv. 2018 Aug;12(4): 601-18.

5 Pouwels S, Hageman D, Gommans LN, Willigendael EM, Nienhuijs SW, Scheltinga MR, et al. Preoperative exercise therapy in surgical care: a scoping review. J Clin Anesth. 2016 Sep;33:476-90.

6 Minnella EM, Awasthi R, Loiselle SE, Agnihotram RV, Ferri LE, Carli F. Effect of Exercise and Nutrition Prehabilitation on Functional Capacity in Esophagogastric Cancer Surgery: A Randomized Clinical Trial. JAMA Surg. 2018 Dec;153(12):1081-9.

7 Piegeler T, Stehr SN, Pfirrmann D, Knödler M, Lordick F, Mehnert A, et al. [Special situations of preconditioning and prehabilitation in oncological visceral surgery]. Chirurg. 2018 Nov;89(11):903-8.

8 Singh F, Galvão DA, Newton RU, Spry NA, Baker MK, Taaffe DR. Feasibility and Preliminary Efficacy of a 10Week Resistance and Aerobic Exercise Intervention During Neoadjuvant Chemoradiation Treatment in Rectal Cancer Patients. Integr Cancer Ther. 2018 Sep;17(3):952-9.

9 Carli F, Charlebois P, Stein B, Feldman L, Zavorsky G, Kim DJ, et al. Randomized clinical trial of prehabilitation in colorectal surgery. Br J Surg. 2010 Aug;97(8):1187-97.

10 Coats V, Maltais F, Simard S, Fréchette E, Tremblay L, Ribeiro F, et al. Feasibility and effectiveness of a homebased exercise training program before lung resection surgery. Can Respir J. 2013 Mar-Apr;20(2):e10-6.

11 Moorthy K, Wynter-Blyth V. Prehabilitation in perioperative care. Br J Surg. 2017 Jun;104(7):802-3.

12 Pfirrmann D, Tug S, Brosteanu O, Mehdorn M, Busse M, Grimminger PP, et al. Internet-based perioperative exercise program in patients with Barrett's carcinoma scheduled for esophagectomy [iPEP - study] a prospective randomized-controlled trial. BMC Cancer. 2017 Jun;17(1):413.

13 van Hagen P, Hulshof MC, van Lanschot JJ, Steyerberg EW, van Berge Henegouwen MI, Wijnhoven BP, et al.; CROSS Group. Preoperative chemoradiotherapy for esophageal or junctional cancer. N Engl J Med. 2012 May;366(22):2074-84. 
Hillen et al.: Use of a Perioperative Web-Based Exercise Program for a Patient with Barrett's Carcinoma Scheduled for Esophagectomy

14 Navidi M, Phillips AW, Griffin SM, Duffield KE, Greystoke A, Sumpter K, et al. Cardiopulmonary fitness before and after neoadjuvant chemotherapy in patients with oesophagogastric cancer. Br J Surg. 2018 Jun;105(7):900-6.

15 Segal R, Zwaal C, Green E, Tomasone JR, Loblaw A, Petrella T, et al.; Exercise for People with Cancer Guideline Development Group. Exercise for people with cancer: a clinical practice guideline. Curr Oncol. 2017 Feb;24(1):40-6.

16 Low DE, Allum W, De Manzoni G, Ferri L, Immanuel A, Kuppusamy M, et al. Guidelines for Perioperative Care in Esophagectomy: Enhanced Recovery After Surgery (ERAS) Society Recommendations. World J Surg. 2019 Feb;43(2):299-330.

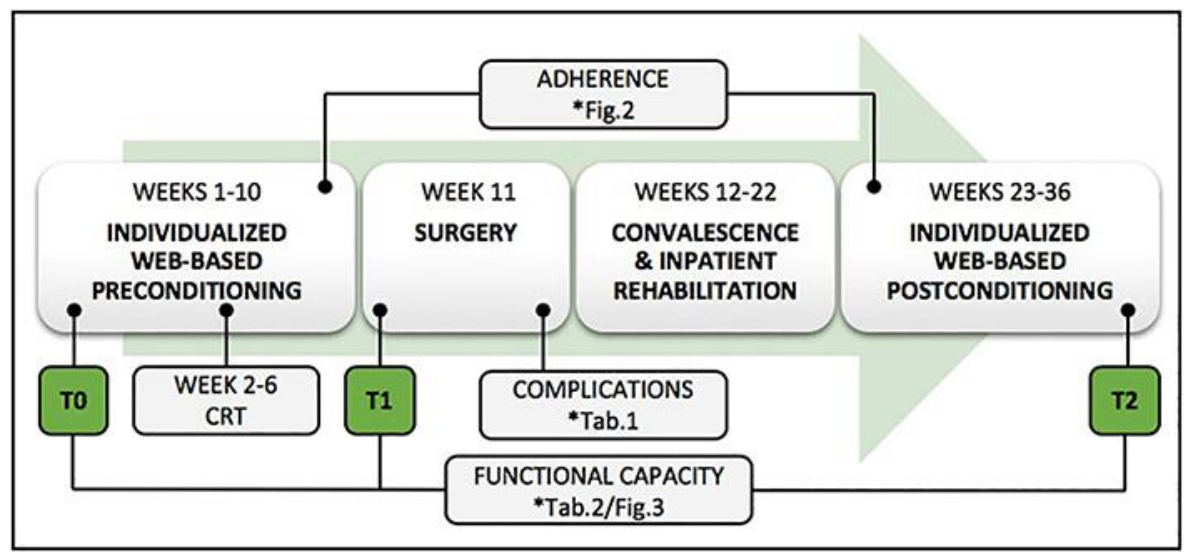

Fig. 1. Overview - interventional course. 


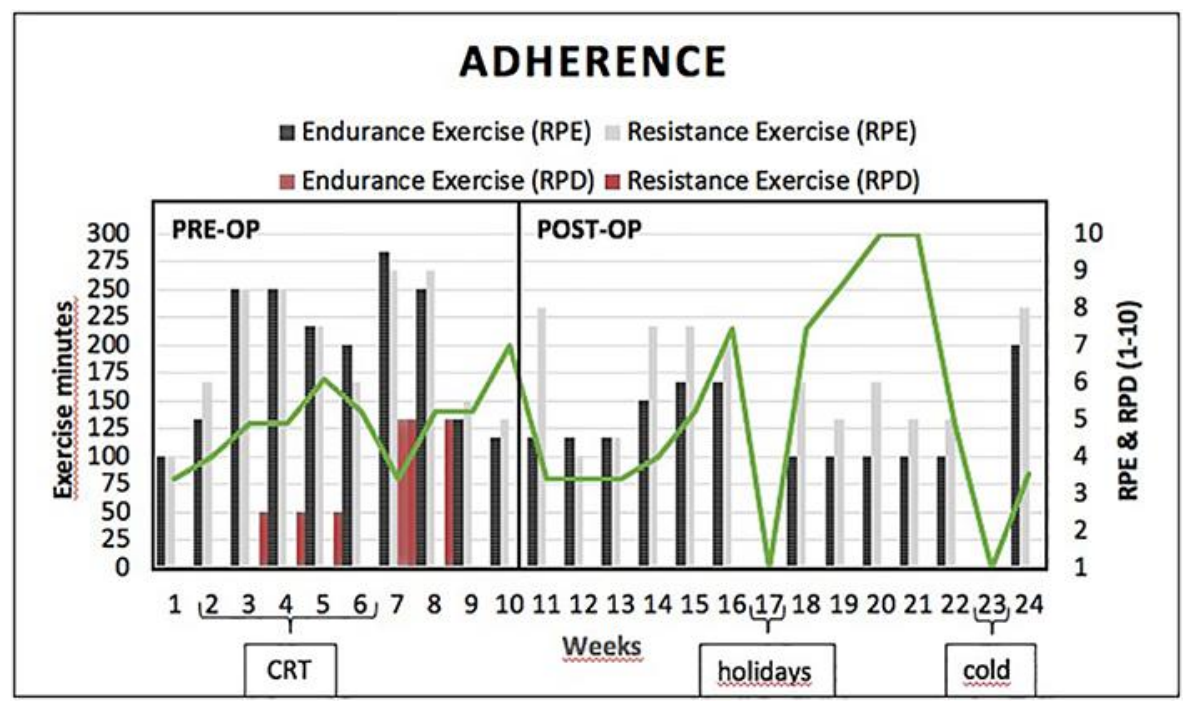

Fig. 2. Overall adherence to the 24 weeks pre- and postconditioning program. Exercise duration (min) depicted as green line and rated intensity (RPE and RPD) depicted as red, grey, and black bars.

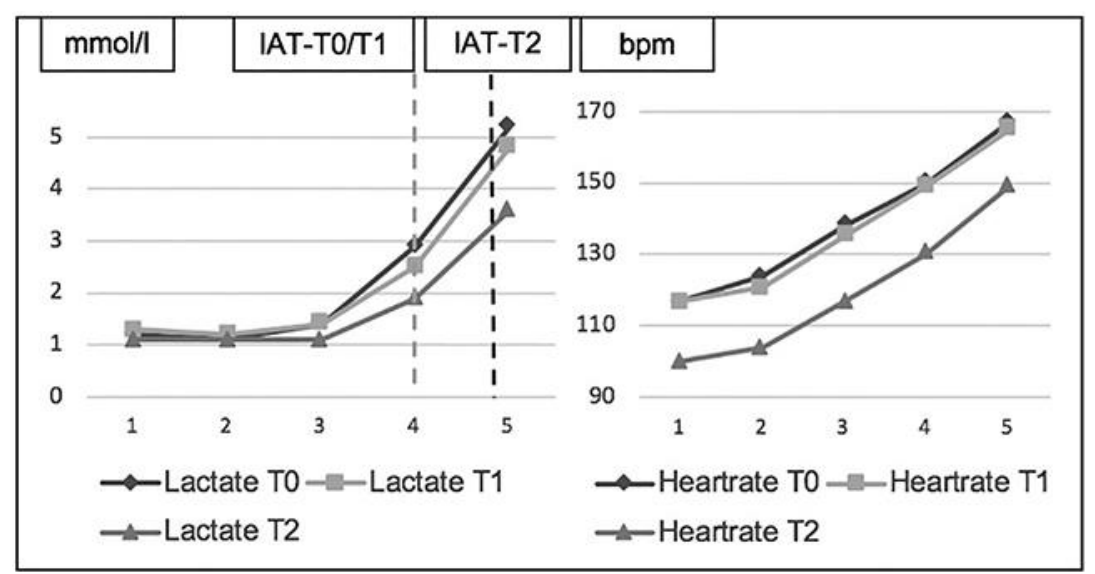

Fig. 3. Blood lactate $(\mathrm{mmol} / \mathrm{L})$, heart rate (bpm) curves (T0-T2) and individual anaerobic threshold (IAT). 
Table 1. Surgery and postoperative complications

Surgery - totally minimally invasive Ivor-Lewis esophagectomy

- Operating time: $330 \mathrm{~min}$

- Anastomosis circular stapler (end-to-side esophagogastrostomy): $25 \mathrm{~mm}$

- Blood loss: $100 \mathrm{~mL}$

- No administration of blood preserves

- Intensive Care Unit care: one day

- Extubated without reintubation

- No relocation

Postoperative complications and reasons for a prolonged hospital stay

- Functional stenosis of the gastric tube with aspiration pneumonia (Therapy (44 days, post surgery:

Endoscopic self-expandable stent in the distal gastric conduit); no anastomotic leak

- Pulmonary: temperature $>38.5<38.9$; diffuse infiltrate in the X-ray image of the lung, corresponding to pneumonia

- Gastric emptying disorder (functional pylorospasm) (Therapy (7 days post surgery): Treatment with endoscopic pylorus dilatation)

Table 2. Descriptive data T0-T2 and Subjective Training evaluation

\begin{tabular}{lllll}
\hline Parameter & T0 & T1 & T2 & Training evaluation \\
\hline Age & 56 & 56 & 57 & I thought the training guidelines were appropriate++ \\
Weight & 90.4 & 90.2 & 80.6 & I wasn't afraid of injuries++ \\
Height & 183 & 183 & 183 & I didn't see any risks during independent training++ \\
BMI & 27 & 26.9 & 24 & I thought the platform was uncomplicated++ \\
HR $_{\text {rest }}$ & 106 & 108 & 106 & I felt the communication as personal++ \\
FEV 1 & 97 & 101 & 92 & \\
FVC & 112 & 110 & 106 & For him, the most important contents were: \\
VEmax $_{\text {m }} /$ min) & 87 & 87 & 78 & - Training support \\
VO $_{2 m a x}(\mathrm{~mL} / \mathrm{min} / \mathrm{kg})$ & 23.1 & 23.2 & 22.2 & - General study participation \\
Increments $(n)$ & 5 & 5 & 5 & - Flexibility through the internet \\
Watts/kgmax & 1.2 & 1.2 & 1.2 & - Individual training planning \\
Watts/kg & 0.9 & 0.9 & 0.9 & - Contact person after inpatient rehabilitation \\
Dysphagia Score & 0 & 0 & 0 & \\
Nutritional Risk Score & 0 & 0 & 0 & Own comment: \\
Stress metabolism Score & 0 & 0 & 0 & "Very happy with the concept" \\
\hline
\end{tabular}

